\title{
AC 2011-1844: TIMELY FEEDBACK AND STRENGTHENED STUDY HABITS VIA COMPUTER AUTOMATED END-OF-LECTURE QUESTIONS
}

\section{Paul J. Weber, Lake Superior State University}

Paul J. Weber is an Assistant Professor at Lake Superior State University. Since completing his Ph.D. at Michigan Tech in 2006, he has taught courses in digital and computer systems as well as electronics, circuit analysis, and robot vision. His research interests include alternative energy, energy efficiency, distributed control, and engineering education. 


\title{
Timely Feedback and Strengthened Study Habits via Computer Automated End-of-Lecture Questions
}

\begin{abstract}
Timely feedback is a critical component of the educational process for both the students and the instructor. Such feedback enables students to gauge how well they are understanding the material and seek help and/or adjust study habits when needed. It also enables the instructor to alter the pace and topics of particular class periods to adjust to the needs of individuals and a class as a whole. Automated End-Of-Lecture Questions (EOLQs) can enable such feedback.

The EOLQs described in this paper consisted of a set of questions that were to be answered prior to the start of the subsequent class period. They were implemented via a Course Management System (CMS). Further individualized feedback for the instructor was made quickly accessible by coupling automatically generated CMS emails with an email client filter.

The assessment of the EOLQs consisted of a survey from two different classes using a 9-value Likert scale as well as one open-ended question about the EOLQs. The results of the survey and question were that the students generally felt that the EOLQs were beneficial to their learning, supplemented traditional homework well, and encouraged them to study more in total and more often. The students were not sure, however, if they were likely to use similar study techniques in future classes. Due to the small sample size $(\mathrm{N}=19)$, these results should be used as a basis for further study rather than being interpreted as generalizable.
\end{abstract}

\section{Introduction}

Timely feedback is crucial to the learning process. Two important goals as an educator therefore are to assess students' comprehension of the covered concepts in a relatively fast manner, and to help students develop study habits which naturally provide ways for them to quickly assess their own understanding. In doing so, students also then learn habits that can help in their learning process to commit concepts to long term memory. Automated End-Of-Lecture Questions (EOLQs) provide one useful tool to achieve these objectives.

This paper will describe how such questions have been implemented via computer automation. The semester-long assignment consisted of one to three questions at the end of the each class period which the students were required to answer prior to the start of the next class period. To do so, students were required to log into a course management system (CMS), within which the questions were posted. For these classes, the chosen CMS was Blackboard, but similar techniques could be used in other CMSs. Immediately after answering the questions, students were provided with the correct response(s) to the question. In addition, the answers were also used as part of a brief recap of that lecture during the next class period, and time was made available for discussion about the questions. 
The remainder of the paper is organized as follows: Section 2 first explains the layout of the EOLQs and the pedagogical basis for their use. Sections 3 and 4 next describe the survey that was utilized to determine their use and student perceptions about them as well as the results of the survey. Section 5 describes possible improvements for subsequent classes as well as potential future research about the use of such EOLQs. Lastly, Section 6 concludes with a summary of the paper and its contributions.

\section{End-of-Lecture Questions Overview and Pedagogical Basis}

As previously mentioned, the EOLQs comprised one to three questions that were assigned after each class to be completed by the students via the CMS prior to the beginning of the next class period. A sample of one of the questions is shown in Figure 1.

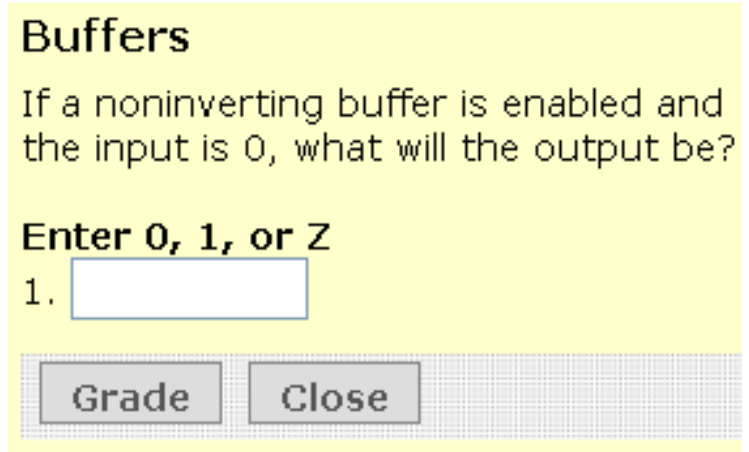

Figure 1: Sample EOLQ

The intended objectives of the EOLQs were threefold:

(1) To encourage the students to commit the concepts discussed in class to long-term memory.

(2) To encourage students to gauge their own understanding of what was discussed in class.

(3) To enable the instructor to gauge the students' understanding of what was discussed in class in a timely manner.

The first objective derives from the need for "formative assessments, which provide students with opportunities to revise and improve the quality of their thinking and understanding." ${ }^{4}$ In many classes, this assessment is implemented via traditional quizzes, exams, and homework problems. Each of these assessment mechanisms were also implemented, but the EOLQs were used as a tool to engage the students in the information in closer proximity to when the topic was first discussed. The hypothesis is that if the same information is seen in class and soon thereafter (ideally later that day), the student is more likely to move the information from short term memory into long term memory.

Sims-Knight and Upchurch state that "informational feedback is effective when there is a clear right or wrong answer, and when the subject is tested immediately following the feedback provision." ${ }^{6}$ Because of this, EOLQs focused on foundational concepts in forms that could be made into true/false, multiple choice, or short answer problems. The need for the use of such questions was also derived from the fact that the questions were automatically graded, which 
would have been significantly more challenging for open-ended questions or those for which many answers exist.

As mentioned previously, the EOLQs were used as a supplement to other assessments. Freeman and McKenzie note that "if the goal is to enhance understanding and applicability of knowledge, it is not sufficient to provide assessments that focus primarily on memory for facts and formulas." ${ }^{4}$ Because of this, traditional paper problem homework and quizzes (as well as exams) were still utilized in the class that involved the application of the foundational concepts in more contextualized and complex manners. This allowed the instructor to better view the students' synthesis and problem solving capabilities.

Fast feedback is critical to student learning, which is where the second (feedback for the students) and third (feedback for the instructor) objectives derive from. The recognition of the need for fast feedback by educators can be seen, for example, in the widespread adoption of technologies such as clickers. ${ }^{2}$ With the capability to automate the feedback, the timeliness of the feedback increases significantly. Whereas items such as clickers enable such feedback in class, CMSs enable similar feedback between subsequent class periods.

An example of the feedback from the EOLQs can be seen in Figure 2. Such feedback enables greater self-regulation in the students learning, which is a critical component of web-based courseware utilization. ${ }^{7}$ It is also important to note that the students not only are provided feedback about whether the answer was correct or incorrect, but also the general reasoning about the problem in the "General Feedback" section. Such specific feedback provides an opportunity to incorporate meta-cognition ${ }^{1,3}$ in which the student can think about his/her reasoning as compared to that of the "General Feedback."

As can also be seen in Figure 2, half of the possible points are given for participation while the other half is allotted based on whether the answer is correct or not. This is intended to provide motivation for the students to try to answer them correctly (looking back at lecture notes where needed, etc.) rather than simply guessing.

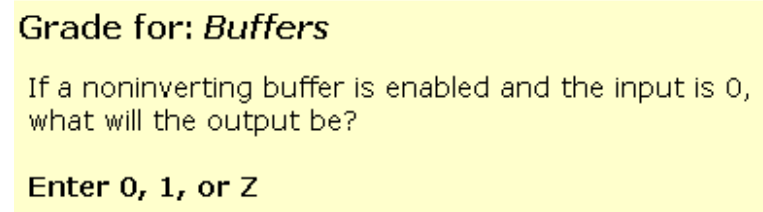

Figure 2: Sample Grade and Feedback for EOLQ 
Lastly, the EOLQs are intended to help the instructor gauge student understanding of the topics that were covered in a more efficient manner (in terms of instructor time). This was achieved by setting up an "email and filter" system. When a student completed a set of EOLQs, the CMS automatically sent the set of responses (from the individual student) to the instructor via email. An email client was then used to filter the responses automatically into a folder. This then enabled the instructor to quickly scroll through individual student responses (in addition to having the usual aggregated statistics on the CMS) prior to the class period. This can be useful both for helping individuals and for examining the responses of the more engaged students as an indicator of what topics might need to be clarified in the upcoming class. This setup thus enabled the instructor the same level of detailed feedback while spending less time overall on creating and grading assignments.

\section{Student Survey and Student Population}

At the end of the semester, students had the option of filling out a survey with a series of 5 questions pertaining to the EOLQs as well as an opportunity to express opinions about them. Each question was a Likert item ${ }^{5}$, for which the students were directed to state the level to which they agreed with the statement. Values ranged from 1 ("Very Strongly Agree") to 9 ("Very Strongly Disagree") in increments of one. The questions with their abbreviations for the results were as follows:

1. Because of the EOLQs, I...Studied more than I would have if there had not been EOLQs. (Studied More)

2. Because of the EOLQs, I...Studied more often (not necessarily more in total) throughout the semester than I would have if there had not been EOLQs. (Studied More Often)

3. I feel that the EOLQs...Were beneficial to my learning. (Beneficial to Learning)

4. I feel that the EOLQs...Supplemented the other homework assignments well. (Supplemented HW Well)

5. I plan to use a study method similar to the EOLQs in future classes (if the teacher does NOT employ similarly structured assignments). (Will Use in Future)

There were 19 students that completed the survey from two engineering classes. One class was a senior-level machine vision course (12 students) while the other was a junior-level digital design course ( 7 students). There were no students that were enrolled in both classes. Furthermore, there was one student from each class that chose not to answer the questionnaire. 


\section{Survey Results}

The results of the survey can be seen in Figure 3. As depicted by the mean of the responses, the students generally thought that they studied more (in total) and more often than they would have had there not been EOLQs. Furthermore, the students agreed with the statements that the EOLQs were beneficial to their learning and supplemented the other homework in the class well. It is important to note, though, that there was no objective measurement of the actual study time, frequency, etc.

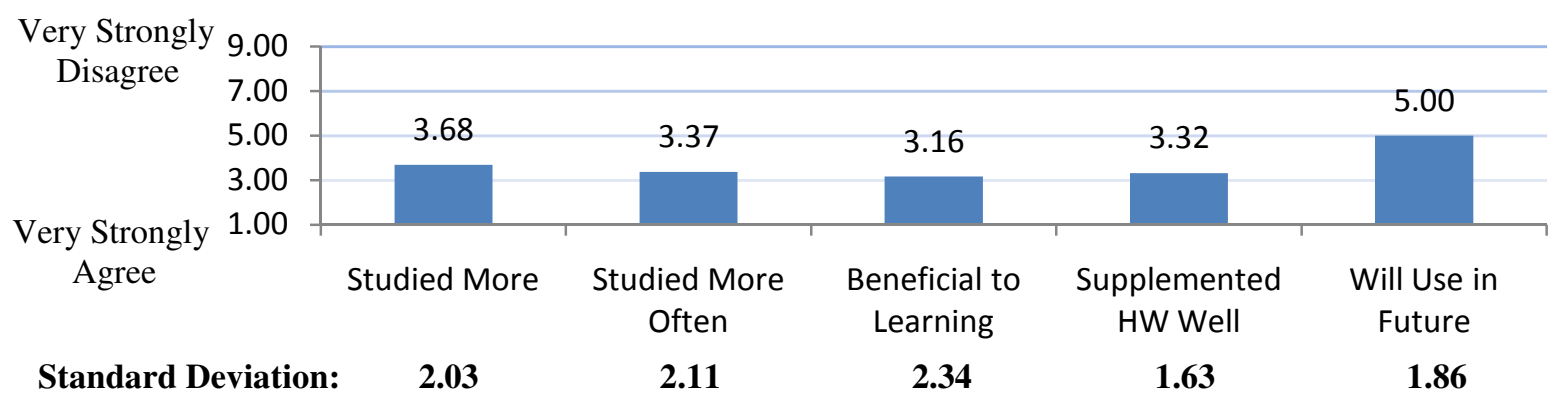

Figure 3: Mean and Standard Deviation of Responses to EOLQ Survey Questions ( $N=19)$

The last column shows the student response to whether they would use a similar study technique in the future. As can be seen, the average of the response was neutral.

Also included in Figure 3 are the standard deviations of the responses, which for the number of respondants could vary anywhere from 0 to just over 4 . These numbers indicated that there was the most consensus that the EOLQs supplemented the homework well while there was the least consensus that they were beneficial to their learning. The histograms of each of the responses can be found in Appendix A.

As indicated in Section 3, the survey also included an opportunity for student comments. These had a broad range, but a sample of them is included below that encapsulates the range of comments:

"I felt that EOLQs were good in the fact that there was work outside of class almost every day that kept new subjects in mind."

"The EOLQs are very helpful, and reinforce class topics well. They are a good idea. However, it would be better if they were posted at the same time every time so they are easier to remember."

"Even though I don't believe the EOLQ's helped (or hindered) my learning, I think that it did help others to not only study, but to know where they were having trouble, to bring it up in class."

"[I] forgot [to complete them] all the time."

“...They were a good study guide."

"I have never had another class with EOLQs.... I think they are a great idea, they are not as burdening as a set of hw assignments but still stimulate and force you to learn something." 
"I feel that the EOLQs were just as effective as more homework assignments would have been. They were actually able to stimulate me more often than the usual homework assignment would have."

In summary, overall the comments reinforced the responses from the other questions. It should be noted that one mechanism that was implemented was to email the class with the subject line "EOLQs Posted" after each class. This was was done based on previous feedback similar to the comment above about forgetting to complete the EOLQs. Making the timing of the posting (e.g. within the first half hour after the class completes) might also help alleviate this issue.

\section{Improvements and Future Research}

One limitation of the work described is that the only assessment of the usefulness of the EOLQs was through a survey. The first aspect of future research should therefore be to more objectively measure their use and effectiveness. For the latter, research could involve a test group of students that complete the EOLQs and a control group would not complete the EOLQs. Pop quizzes could then be given (for which student's would not have the opportunity to study for) to see how much gain in retention there is between those that use the EOLQs versus those that use only traditional homework. This would enable the study to have a reasonable "construct validity" (i.e. it would be a reasonable measure of the EOLQs' effectiveness without the interaction of other variables, such as studying for the quiz).

Another hope for the EOLQs was that students would adjust their study habits in the future to better align them with the idea of studying more often rather than cramming in study sessions prior to large assessments. Since the results of the survey indicated that this likely did not happen, it would be useful to inquire about the obstacles to incorporating EOLQ-like study habits. If repeating the assessment about whether study habits did change, one could also use GPA or major-GPA to try to control the effects of already learned study habits or aptitude for the course material.

The last area that would be useful to gauge was whether the students read the "General Feedback" and furthermore if they considered how it either aligned or did not align with their own thinking.

\section{Summary}

In conclusion, the EOLQs did provide a quicker feedback tool for the instructor as compared to assessments of similar learning depth. They also provided more individualized feedback as compared to the typical aggregated scores of the CMS and enabled the instructor to better tailor the next class's discussions to the needs of the students. Students felt that the EOLQs were useful. However, they were unsure whether they would implement similar methods on their own in the future. As a result, it will be important to extend this work to determine the obstacles to study habit adjustment and to more objectively assess the effectiveness on long-term retention of material with larger student populations to enable more generalizable results. 


\section{References}

1. Brown, A.L. 1980. Metacognitive development and reading. In Theoretical issues in reading comprehension: Perspectives from cognitive psychology, linguistics, artificial intelligence and education, eds. R.J. Spiro, B.C. Bruce, and W.F. Brewer. Hillsdale, NJ: Lawrence Erlbaum.

2. Chen, J.C., Whittinghill, D.C., and Kadlowec, J.A. 2010. Classes that click: fast, rich feedback to enhance student learning and satisfaction. Journal of Engineering Education, 99(2): 159 - 168.

3. Flavell, J.H. 1991. Understanding memory access. In Cognition and the symbolic processes: Applied and ecological perspectives, eds. R. Hoffman and D. Palermo. Hillsdale, NH: Lawrence Erlbaum.

4. Freeman, M., and McKenzie, J. 2001. Aligning peer assessment and peer learning for large classes: The case for an online self and peer assessment system. In Peer learning in higher education, eds. D. Boud, R. Cohen, and J. Sampson, 156 - 169. London: Kogan Page Limited.

5. Likert, R. (1932). A technique for the measurement of attitudes. Archives of Psychology, 140, 1-55.

6. Sims-Knight, J., and Upchurch, R.L. 2001. What's wrong with giving students feedback. In Proceedings of the 2001 American Society of Engineering Education Annual Conference and Exposition. Albequerque, NM.

7. Steif, P.S., Dollar, A. 2009. Study of usage patterns and learning gains in a web-based interactive static course. Journal of Engineering Education, 98(4): 321 - 333. 


\section{Appendix A: Histograms of Student Responses}

This appendix comprises the histograms of the student responses to the five questions about the EOLQs.

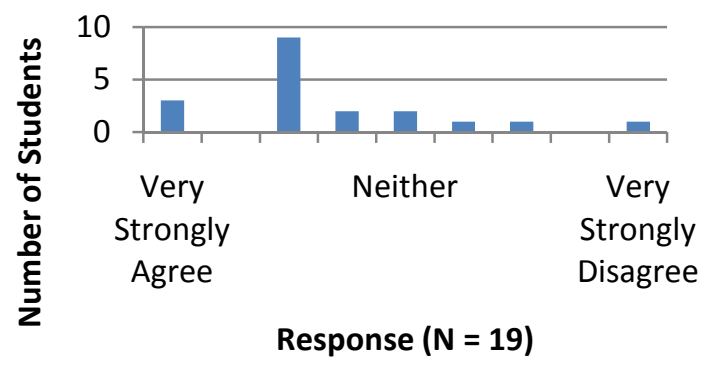

Figure A-1: Response to Whether the Student Studied More Because of the EOLQs

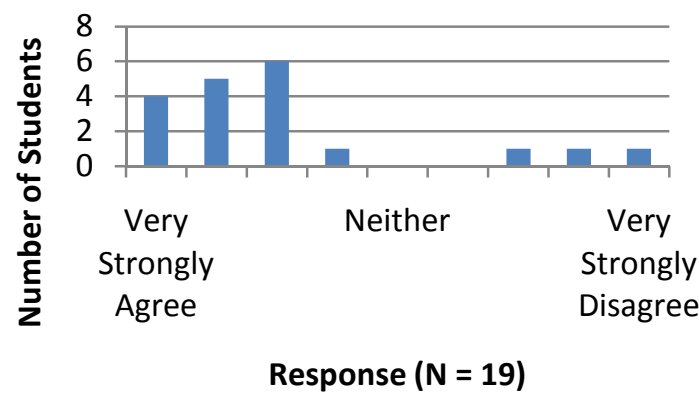

Figure A-3: Response to Whether the Student Felt the EOLQs Were Beneficial to His/Her Learning

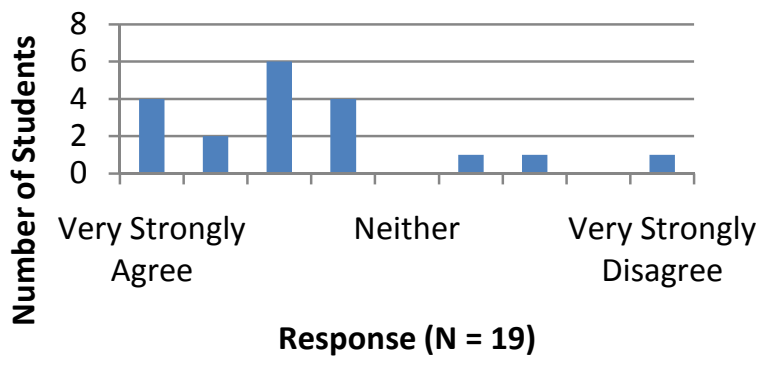

Figure A-2: Response to Whether the Student Studied More Often Because of the EOLQs

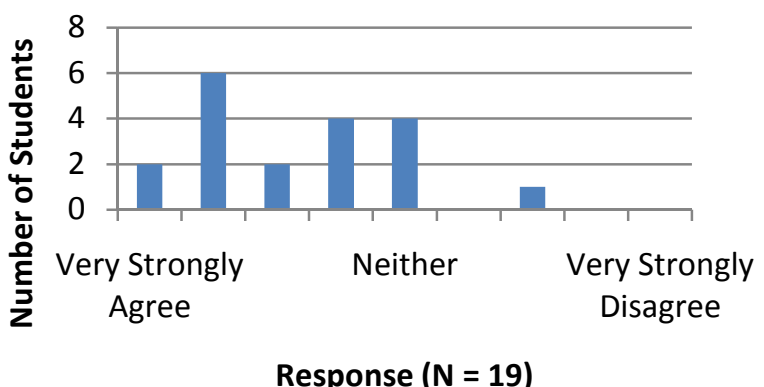

Figure A-4: Response to Whether the Student Felt the EOLQs Supplemented the Other Class Homework Well

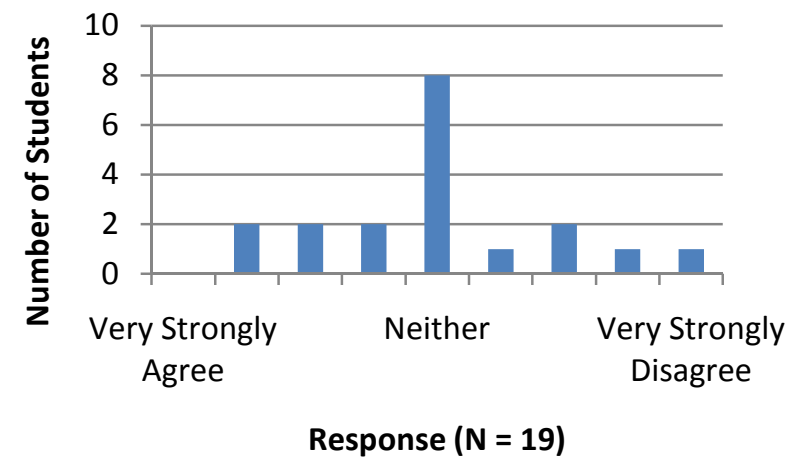

Figure A-5: Response to Whether the Student Thought That S/he Would Use Similar Techniques to the EOLQs to Study in Future Classes 